\title{
Two-Dimensional Solitons on the Surface of Magnetic Fluids
}

\author{
Reinhard Richter $^{1}$ and I. V. Barashenkov ${ }^{2}$ \\ ${ }^{1}$ Experimentalphysik V, Universität Bayreuth, D-95440 Bayreuth, Germany \\ ${ }^{2}$ University of Cape Town, Rondebosch 7700, South Africa \\ (Received 29 July 2004; published 11 May 2005)
}

\begin{abstract}
We report an observation of a stable solitonlike structure on the surface of a ferrofluid, generated by a local perturbation in the hysteretic regime of the Rosensweig instability. Unlike other pattern-forming systems with localized 2D structures, magnetic fluids are characterized by energy conservation; hence their mechanism of soliton stabilization is different from the previously discussed gain-loss balance mechanism. The radioscopic measurements of the soliton's surface profile suggest that locking on the wavelength defined by the nonmonotonic dispersion curve is instrumental in its stabilization.
\end{abstract}

\section{DOI: 10.1103/PhysRevLett.94.184503}

To date, stable solitary waves have been experimentally observed in a variety of one-dimensional and quasi-onedimensional physical systems. In 2D, dispersive nonlinear systems are prone to collapse instabilities and hence the 2D solitons turned out to be more elusive. (Here we use the term "soliton" in a broad physical sense, as a synonym of localized structure.) So far, the list of experimentally detectable 2D localized objects was confined mostly to vortices in superfluids, superconductors, and other media on one hand, and dissipative solitons in nonequilibrium systems on the other. While the stability of the former is due to their nontrivial topology, the latter come into being via the balance of strong dissipation and energy gain. Examples include current filaments in gas discharge systems [1], oscillons in fluids and granular materials [2], breathing spots in chemical reactions [3], and feedback and cavity solitons in optics [4]. Despite some encouraging theoretical insights, the question of whether 2D nontopological solitons can arise in conservative systems has remained open.

In this Letter we report an experimental observation of a strongly localized, stable stationary soliton on the surface of magnetic fluid (MF) in a stationary magnetic field. MF is a dispersion of magnetic nanoparticles, and thus has a high relative permeability $\mu_{\mathrm{r}}$ [5]. This is a lossless system; a horizontal layer of MF in a vertically applied magnetic induction $B$ is characterized by the energy density $[6,7]$ :

$$
\begin{aligned}
\mathcal{F}[h(x, y)]= & \frac{\rho g}{2} h^{2}(x, y)-\int_{0}^{h} d z B \frac{\mu_{\mathrm{r}}-1}{2} H_{\mathrm{MF}}(x, y, z) \\
& +\sigma \sqrt{1+\left[\partial_{x} h(x, y)\right]^{2}+\left[\partial_{y} h(x, y)\right]^{2}} .
\end{aligned}
$$

Here $\rho$ and $\sigma$ are the density and surface tension of the MF, $h(x, y)$ the local height of the liquid layer, and $H_{\mathrm{MF}}(x, y, z)$ is the magnetic field in the presence of the MF. The three terms in Eq. (1) represent the hydrostatic, magnetic, and surface energy, respectively. As the surface profile deviates from the flat reference state, the first and last term grow whereas the magnetic energy decreases. For sufficiently large $B$, this gives rise to the normal field, or Rosensweig, instability $[5,8]$.
PACS numbers: 47.65. $+\mathrm{a}, 47.60 .+\mathrm{i}, 47.62 .+\mathrm{q}$

Our experimental setup is sketched in Fig. 1. A Teflon ${ }^{\circledR}$ vessel with the radius $R=60 \mathrm{~mm}$ and depth of $3 \mathrm{~mm}$ [9] is filled with MF up to the brim and placed on the common axis midway between two Helmholtz coils. An x-ray tube is mounted above the center of the vessel at a distance of $1606 \mathrm{~mm}$. The radiation transmitted through the fluid layer and the bottom of the vessel is recorded by an x-ray sensitive photodiode array detector (16 bit) connected to a computer. The full surface relief is then reconstructed from the calibrated radioscopic images. For details, see [10]. The experiments were performed with the magnetic fluid EMG 901, Lot F121901 AX from Ferrotec. Its material parameters have been measured to $\mu_{\mathrm{r}}=3.2, \rho=$ $1.406 \mathrm{~g} \mathrm{~cm}^{-3}$, and $\sigma=25 \pm 0.7 \mathrm{mN} / \mathrm{m}$.

Starting from a flat layer at $B=0 \mathrm{mT}$, we increase the external induction adiabatically in steps of $15 \mu \mathrm{T}$, pausing for $30 \mathrm{~s}$ after each increase. As shown in Fig. 2(a), a deformation of the surface of the liquid appears first at the edge of the vessel. This is due to the magnetic field gradient induced by the discontinuous magnetization at the edge of the liquid layer. Increasing the induction further gives rise to a fully developed pattern of the Rosensweig instability, as shown in Fig. 2(b) and 2(c).

We measured the top-to-bottom height $A$ of the stationary fluid pattern arising in the adiabatic increase and decrease of $B$. To avoid the edge-induced imperfections

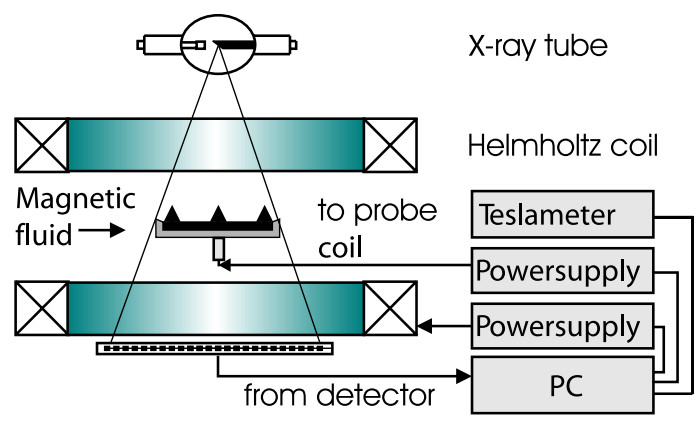

FIG. 1 (color online). Sketch of the experimental setup. See text for details. 

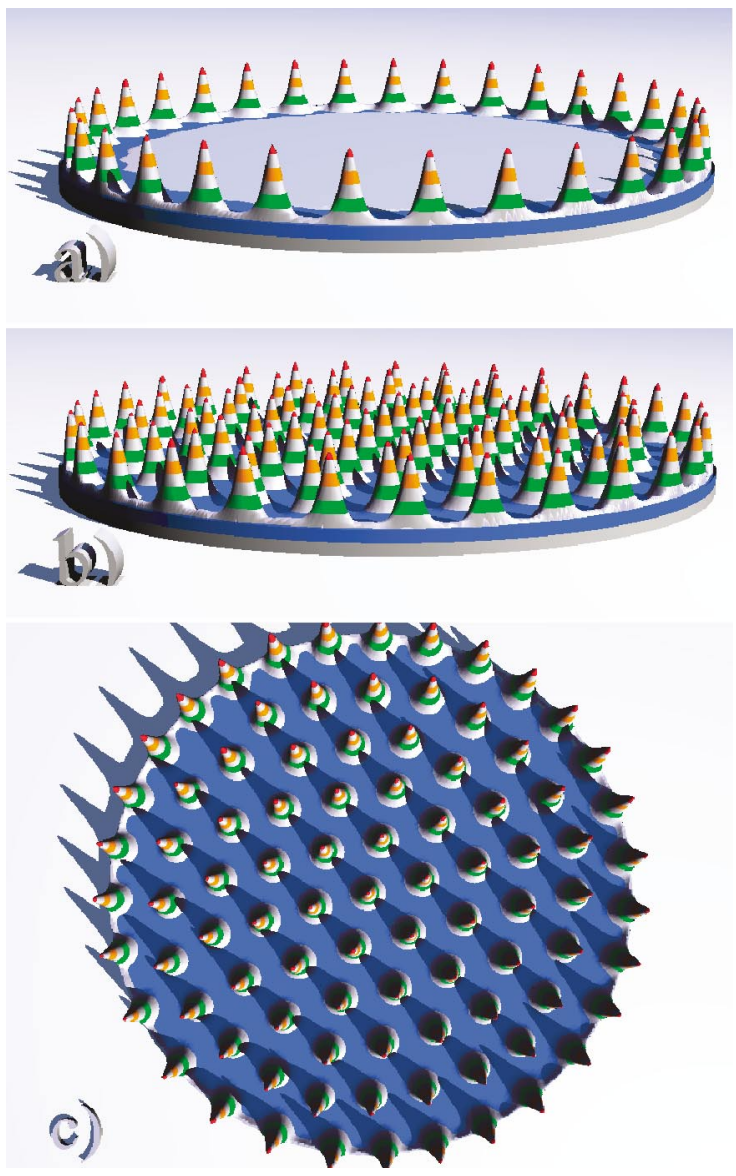

FIG. 2 (color). Surface reliefs as reconstructed from the radioscopic images for (a) $B=8.922 \mathrm{mT}$, and (b), (c) $10.407 \mathrm{mT}$. Each color indicates a layer thickness of $1 \mathrm{~mm}$.

in the character of the bifurcation, we only consider spikes located within $11 \mathrm{~mm}$ from the center of the dish. Figure 3 displays results obtained for 400 values of $B$. As $B$ is increased, a sudden transition to the upper branch occurs at $B_{c}=9.025 \mathrm{mT}$. For $B>B_{c}$, the entire surface is covered by a lattice of liquid spikes, which is hexagonal away from the boundary. Decreasing $B$, the order parameter $A$ remains on the upper branch all the way to $B^{*}=8.076 \mathrm{mT}$ where it drops to the flat reference level. Thus the diagram shows a subcritical bifurcation to hexagons. The solid and dashed lines display a fit to the roots of the corresponding amplitude equation of Ref. [7].

To study the stability of the flat surface to local perturbations (in the hysteretic regime), a small air coil with the inner diameter of $8 \mathrm{~mm}$ was placed under the center of the vessel (see Fig. 1). This allows to increase, locally, the magnetic induction. A local pulse of $B_{+}=0.68 \mathrm{mT}$ added to the uniform field of $B=8.91 \mathrm{mT}$ produces a single stationary spike of fluid, surrounded by a circular dip, which does not disperse after $B_{+}$has been turned off. Figure 4 presents a measured relief of this radially symmetric state which will be referred to as the soliton. The soliton is a stable nondecaying structure; it remained intact for days. After its formation at the center of the dish, the soliton was

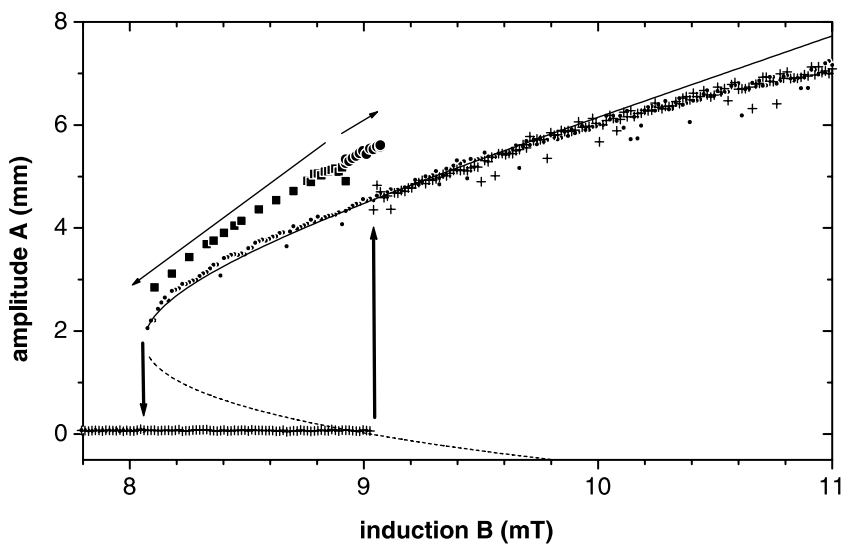

FIG. 3. The amplitude of the pattern for $r<11 \mathrm{~mm}$ vs the magnetic induction. The crosses (dots) mark the values for increasing (decreasing) induction, respectively. The solid (dashed) lines display the least square fit to the roots $A_{ \pm}=$ $\left[\gamma(1+\epsilon) \pm \sqrt{\gamma^{2}(1+\epsilon)^{2}+4 \epsilon g}\right] /(2 g)$, of the amplitude equation $\epsilon A+\gamma(1+\epsilon) A^{2}-g A^{3}=0$ of Ref. [7] with $\gamma=$ $0.281 \mathrm{~mm}^{-1}$ and $g=0.062 \mathrm{~mm}^{-2}$. The full circles (squares) give the amplitude of the localized spike initiated at $B=$ $8.91 \mathrm{mT}$ for increasing (decreasing) induction, respectively.

often seen to float around (with $v \sim 0.1 \mathrm{~mm} / \mathrm{s}$ ), until reaching an equilibrium position somewhere near the edge of the dish. This behavior can be attributed to radial gradients of the magnetic field due to the discontinuous magnetization at the edge and the ring of spikes pinned along the perimeter of the dish.

We examined the range of stability of the soliton generated by a pulse with $B_{+}=0.68 \mathrm{mT}$ added to the uniform induction $B=8.91 \mathrm{mT}$. Reducing $B$ adiabatically, we measured the corresponding amplitude of the soliton (marked by full squares in Fig. 3). Similarly to the spikes in the hexagonal pattern, the height of the soliton decreases as $B$ is reduced. The soliton decays for $B<8.09 \mathrm{mT}$, which is close to $B^{*}=8.076 \mathrm{mT}$, the lower stability boundary of the hexagonal pattern. As $B$ is increased, the amplitude of the soliton grows, as indicated in Fig. 3 by full circles. At $B=9.055 \mathrm{mT}$, a sudden transition from the soliton to the fully developed Rosensweig pattern occurs. This value is somewhat larger than $B_{c}=9.025 \mathrm{mT}$; this is due to the fact that the birth of the soliton produces a slight

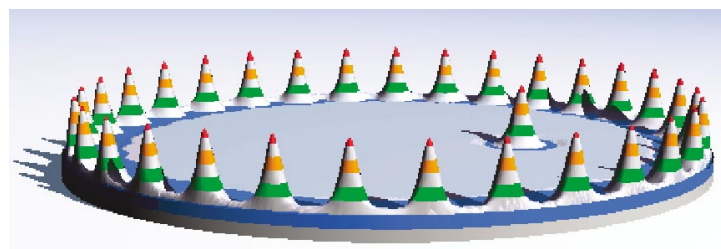

FIG. 4 (color). A single soliton surrounded by the unperturbed magnetic liquid. The magnetic induction generated by the Helmholtz coils amounts to $B=8.91 \mathrm{mT}$. The amplitude of the local pulse which produced the soliton was $B_{+}=0.68 \mathrm{mT}$ at the bottom of the vessel. 
drop in the flat layer thickness, which shifts $B_{c}-$ similarly to Fig. 5 in Ref. [7].

In order to illustrate the robustness of the soliton's shape we show in Fig. 5 the azimuthally averaged profiles of two different solitons, produced in two separate experiments at $B=9.07 \mathrm{mT}$. The profiles are practically indistinguishable. Also plotted are two half periods of the corresponding hexagonal lattice. In agreement with Fig. 3, the soliton is about $1 \mathrm{~mm}$ taller than the spikes of the lattice. This may be attributed to the fact that the spikes emerge simultaneously, and thus have to share the liquid available. However, the width of the soliton is exactly equal to the period of the lattice. Therefore, there is a preferred wavelength in the system, defined by the lattice, and the soliton locks to this length. As we show now, this width locking is the central part of the soliton's stabilization mechanism.

Consider the dispersion relation of a semi-infinite layer of inviscid MF [8],

$$
\omega^{2}=g k-\mu_{0} \mu_{r} \frac{\left(\mu_{r}-1\right)^{2}}{\mu_{r}+1} \frac{1}{\rho} H^{2} k^{2}+\frac{\sigma}{\rho} k^{3} .
$$

Here $\omega$ is the frequency, $k=|\vec{k}|$ the wave number, $H$ the strength of the external magnetic field, and $\mu_{0}$ the magnetic field constant. While the first and the third terms account for the gravity and capillary effects and are common for all fluids, the second term is specific just for the MF. As $H$ is increased above $H_{c}$, where $H_{c}^{2}=\frac{2\left(\mu_{r}+1\right)}{\mu\left(\mu_{r}-1\right)^{2}} \times$ $(\rho g \sigma)^{1 / 2}$, a band of wave numbers with $\omega^{2}<0$ appears around $k_{c}=(\rho g / \sigma)^{1 / 2}$ and the flat state loses its stability to the hexagonal pattern. Since the soliton should decay to the flat surface as $\vec{x}^{2} \rightarrow \infty$, there can be no stable solitons for $H>H_{c}$.

Let now $H<H_{c}$. On the qualitative level, our system can be modeled by a conservative analogue of the SwiftHohenberg equation:

$$
\ddot{u}+\left(k_{0}^{2}+\nabla^{2}\right)^{2} u+a u=3 b u^{2}-2 c u^{3} ; \quad b, c>0 .
$$

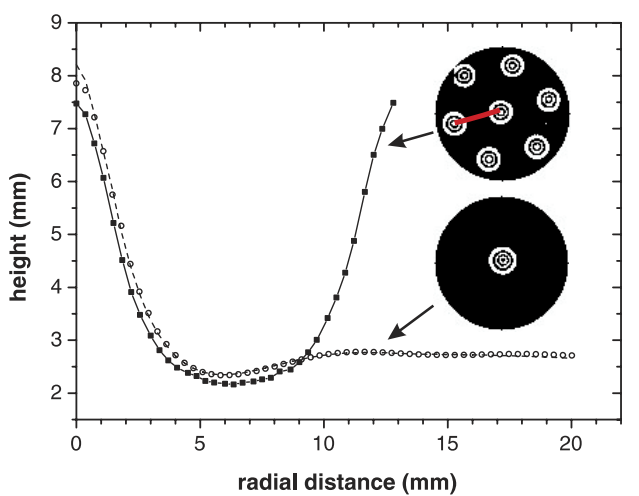

FIG. 5 (color online). The filled squares mark the profile of one period of the hexagonal pattern, measured at $B=9.07 \mathrm{mT}$ in the center of the vessel; $r<8.8 \mathrm{~mm}$. Azimuthally averaged height profiles of two different solitons, measured at the same induction, are depicted by open symbols (one) and a dashed line (the other).
Equation (3) can be used as a model since (a) it has a nonmonotonic dispersion relation $\omega^{2}=a+k_{0}^{4}-2 k_{0}^{2} k^{2}+$ $k^{4}$, where, as in (2), the destabilizing $k^{2}$ term is opposed by a higher power of $k$; and (b) it has a symmetry-breaking hysteretic nonlinearity which was shown to provide a fairly accurate approximation of the amplitude of the hexagonal pattern [7] (see Fig. 3). We have verified, numerically, that Eq. (3) does indeed have a stable stationary radially symmetric soliton solution coexisting with stable hexagons in a broad parameter range. Its stability can be explained by a Derrick-type argument for the corresponding energy functional,

$$
E=\int\left[\dot{u}^{2}+V(u)-2 k_{0}^{2}(\nabla u)^{2}+\left(\nabla^{2} u\right)^{2}\right] d^{2} x
$$

Here $V(u)=\left(a+k_{0}^{4}\right) u^{2}-2 b u^{3}+c u^{4}$. A scaling perturbation $u(\vec{x}) \rightarrow u(\kappa \vec{x})$ takes the stationary energy to

$$
\begin{aligned}
E\left(\kappa^{2}\right)= & \frac{1}{\kappa^{2}} \int V d^{2} x-2 k_{0}^{2} \int(\nabla u)^{2} d^{2} x \\
& +\kappa^{2} \int\left(\nabla^{2} u\right)^{2} d^{2} x .
\end{aligned}
$$

The first term (nonlinearity) opposes the dispersive broadening of the soliton (for which $\kappa \rightarrow 0$ ) while the last one prevents the nonlinear blowup (for which $\kappa \rightarrow \infty$ ). In a similar way, the first and last term in (2) make contributions to the energy which scale as $\kappa^{-1}$ and $\kappa$, respectively. The first term (along with the nonlinearity) opposes the spreading and the last one arrests the blowup. Next, setting the derivative $\left(d E / d \kappa^{2}\right)_{\kappa=1}$ to zero gives $\int V d^{2} x=$ $\int\left(\nabla^{2} u\right)^{2} d^{2} x$. Using this relation, the second derivative, $d^{2} E / d\left(\kappa^{2}\right)^{2}$, is calculated to be $2 \int\left(\nabla^{2} u\right)^{2} d^{2} x>0$, which means that the soliton renders the energy minimum. If Eq. (3) did not include the higher-derivative term, the energy would not have a nontrivial minimum. The introduction of a higher derivative (or, equivalently, the nonmonotonicity of the dispersion curve) sets a preferential wavelength in the system - to which the soliton locks and stabilizes. A similar stabilization mechanism was dis-

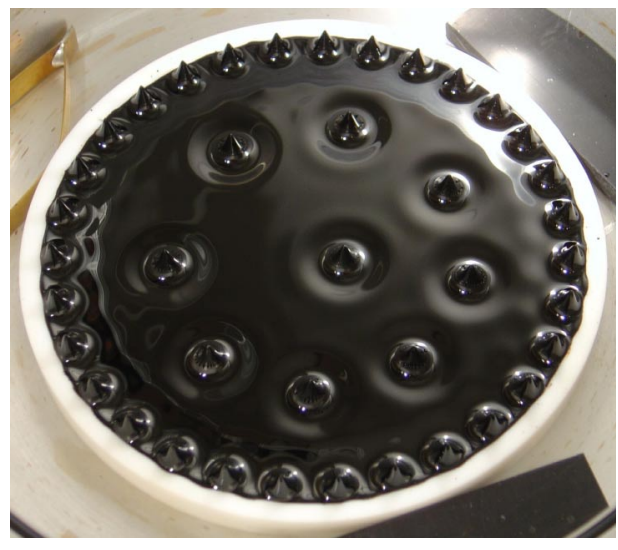

FIG. 6 (color online). Nine solitons at $B=8.91 \mathrm{mT}$. 


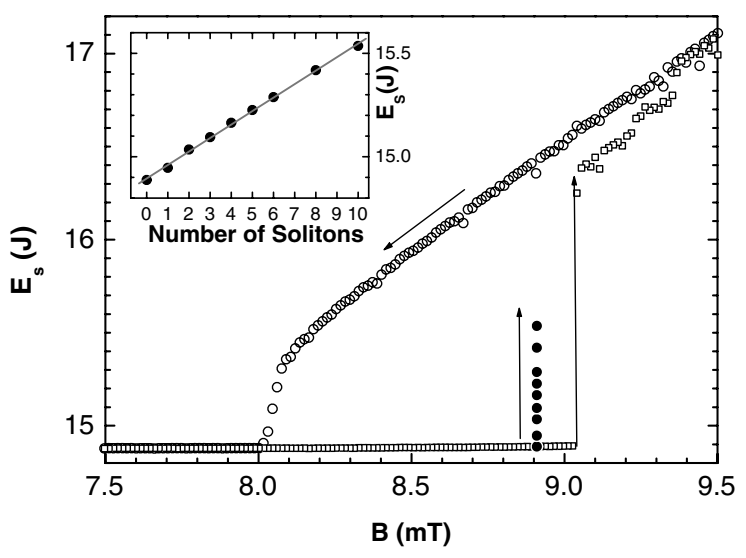

FIG. 7. The surface energy of the liquid layer for increasing (open squares) and decreasing (circles) magnetic induction. The full circles mark the increase of $E_{\mathrm{s}}$ through the successive generation of solitons at $B=8.91 \mathrm{mT}$ (see also inset). To reduce the influence of the perimeter spikes, only the area $r<0.88 R$ was covered, where $R$ is the radius of the vessel.

cussed before in the context of the wave front locking [11]; see also [12].

Applying, repeatedly, pulses of $B_{+}$and allowing the newly born solitons to drift away from the site of the probe coil, we were able to generate two, three, and more solitons. Figure 6 presents an example of a 9-soliton configuration, with only one remaining at the center. In this way, it is possible to increase the surface energy of the liquid layer in steps. This is illustrated in Fig. 7 which also shows the surface energy of the Rosensweig pattern as a hysteretic function of $B$. Thus, one can reach the region between the two branches of this function which is not accessible for the standard Rosensweig instability.

Could solitons serve as building blocks in the formation of periodic patterns? We have observed that, if additional care is taken to suppress the edge-induced inhomogeneity of the magnetization, solitons can form moleculelike clusters (Fig. 8). This may seem to contradict the repulsive nature of the dipole-dipole interaction; however, there is a simple mechanism that can account for the binding. Indeed, each soliton is surrounded by concentric dips representing ringlike regions of depleted magnetic induction. The innermost, deepest ring is clearly visible in Figs. 4-6; a higher-resolution measurement allows to discern another, shallower dip of larger radius. The dips create a potential relief which may capture the partner soliton(s). As the density of solitons grows, the multisoliton cluster evolves towards the hexagonal Rosensweig lattice. It still remains to be understood whether the cluster-lattice transition requires additional excitation energy.

In conclusion, we found stable 2D solitons on the surface of a ferrofluid in the hysteretic regime of the Rosensweig instability. These objects are easy to generate and control and they are easily set in motion; this opens ways for studying their binding and scattering. Because of the conservative nature of the ferrosolitons, and unlike the

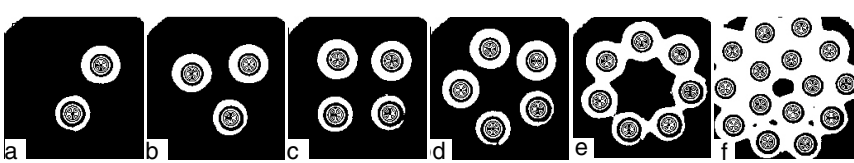

FIG. 8. The solitons can form moleculelike clusters: (a) di-, (b) tri-, (c) tetra-, (d) penta-, (e) hepta-, and (f) oligomers. The height is indicated by switching between black and white after each $\mathrm{mm}$. Here $B=8.71 \mathrm{mT}$; each panel covers the area of $(87 \mathrm{~mm})^{2}$.

localized structures observed previously in dissipative systems, the balance of dissipation and energy gain plays no role in their stabilization. Instead, we suggest a stabilization mechanism which appeals to the locking of the soliton to the wavelength imposed by the nonmonotonic dispersion relation. This mechanism can also be at work in other conservative systems with preferred wavelengths, e.g., in electrostatics and elasticity [13].

We thank I. Rehberg, W. Pesch, and R. Friedrich for discussions and K. Staliunas for providing a code for Eq. (3). Support by NRF of South Africa, Grant No. 205723, and Deutsche Forschungsgemeinschaft, Grants HBFG 051-201 and $\mathrm{Ri} 1054 / 1-4$ is gratefully acknowledged.

[1] Yu. A. Astrov and Yu. A. Logvin, Phys. Rev. Lett. 79, 2983 (1997); I. Müller, E. Ammelt, and H.-G. Purwins, ibid. 82, 3428 (1999).

[2] P. B. Umbanhowar, F. Melo, and H. L. Swinney, Nature (London) 382, 793 (1996); O. Lioubashevski, H. Arbell, and J. Fineberg, Phys. Rev. Lett. 76, 3959 (1996); O. Lioubashevski et al., ibid. 83, 3190 (1999); H. Arbell and J. Fineberg, ibid. 85, 756 (2000); D. Astruc and S. Fauve, in Fluid Mechanics and Its Applications (Kluwer, Dordrecht, 2001), Vol. 62, pp. 39-46.

[3] D. Haim et al., Phys. Rev. Lett. 77, 190 (1996); V. K. Vanag and I. R. Epstein, ibid. 92, 128301 (2004).

[4] V.B. Taranenko, K. Staliunas, and C.O. Weiss, Phys. Rev. A 56, 1582 (1997); B. Schäpers et al., Phys. Rev. Lett. 85, 748 (2000); S. Barland et al., Nature (London) 419, 699 (2002).

[5] R.E. Rosensweig, Ferrohydrodynamics (Cambridge University Press, Cambridge, England, 1985).

[6] A. Gailitis, J. Fluid Mech. 82, 401 (1977).

[7] R. Friedrichs and A. Engel, Phys. Rev. E 64, 021406 (2001).

[8] M. D. Cowley and R. E. Rosensweig, J. Fluid Mech. 30, 671 (1967).

[9] B. Reimann, R. Richter, I. Rehberg, and A. Lange, Phys. Rev. E 68, 036220 (2003).

[10] R. Richter and J. Bläsing, Rev. Sci. Instrum. 72, 1729 (2001).

[11] Y. Pomeau, Physica D (Amsterdam) 23, 3 (1986).

[12] H. Sakaguchi and H. R. Brand, Europhys. Lett. 38, 341 (1997).

[13] G. I. Taylor and A. D. McEwan, J. Fluid Mech. 22, 1 (1965); G. J. Stroebel and W.H. Warner, J. Elast. 3, 185 (1973). 